\title{
Low-Frequency Response of Ptychography in the TEM.
}

\author{
Daniel J. Johnson ${ }^{1}$ and Andrew M. Maiden ${ }^{1}$. \\ 1. Department of Electronic and Electrical Engineering, University of Sheffield, S1 3JD, UK.
}

In the past 10 years, ptychography has emerged as a practical phase-imaging technique in the transmission electron microscope (TEM). Ptychography is a coherent diffractive imaging (CDI) technique that operates by translating a specimen relative to a localised illumination 'probe', and capturing the resulting diffraction patterns. The probe is shifted to a number of positions across the specimen, such that each new probe position overlaps with the previous ones by some specified amount. Capturing diffraction data from overlapping regions in this way creates a redundancy of information that allows algorithmic reconstruction of the complete exit wavefront formed by the probe and specimen.

A recent report of ptychography in the TEM, using a selected-area aperture to form the probe, suggested a problem with the response of the technique to low-frequency phase information [1]. Although the highfrequency response of ptychography has been well documented through investigation of the resolution limit of the technique, there has been little report on the low-frequency capabilities. We investigated, via simulation, the influence of the effective propagation distance on the reconstruction of low-frequency phase features in a TEM selected-area ptychography (SAP) experiment. This paper presents the lowfrequency performance of ptychography as a function of the effective propagation distance.

The object was simulated as soft-edged phase disc, $820 \mathrm{~nm}$ in diameter, with a linear phase gradient across its surface, on a flat (zero phase) field (Fig. 1). This object was selected as the simplest example of an object featuring a large region of low-frequency phase information, whilst still being recoverable through ptychographic reconstruction. The diffraction pattern data set was created by complex multiplication of this object with the recovered probe function from a previous SAP experiment (effective probe diameter at the object of $208 \mathrm{~nm}$ ) [1]. The probe function was shifted relative to the object function to 400 positions in a pseudo-raster grid. This was created by taking a $20 \times 20$ raster grid, with separations equivalent to $20 \%$ of the probe extent, and applying a random offset to each position (with a maximum offset magnitude equal to $20 \%$ of the position spacing) to prevent the formation of periodic artefacts in the reconstruction.

16 separate diffraction pattern sets were collected, each with a different Fresnel number, $F=a^{2} / \lambda z$, where the simulated propagation distance, $z$, had a different value for each set from the range $1.0 \mathrm{e}-3 \leq z \leq 1.6 \mathrm{e}$ $2 \mathrm{~m}$; the wavelength of the illuminating radiation, $\lambda=2.5079 \mathrm{e}-12 \mathrm{~m}$, corresponded to electrons accelerated through $200 \mathrm{kV}$ in the TEM; and the radius of the simulated probe, $a=104 \mathrm{~nm}$, was taken from [1]. This gave a range $0.3 \leq F \leq 2.9$, ensuring the validity of the Fresnel propagator.

The reconstructions were performed using the ptychographic iterative engine (PIE), using an object update function from [2] with update parameter $\alpha=0.5$. The normalised error in the ptychographic reconstruction was tracked using the 'ePIE' error metric [3]. The algorithm was cycled for a maximum of 500 iterations, or until the normalised error value decreased to 1.0e-8. Experiments were initialised with the probe function used to create the diffraction patterns as the probe estimate, and a free-space field for the object estimate.

Our results suggest a relationship between increasing propagation distance and decreasing convergence 
time for ptychographic experiments on low-frequency phase objects in the Fresnel region (Fig. 2). This is likely due to the nature of diffraction pattern formation by a low-frequency phase object - for diffraction patterns formed when the probe is entirely within the ramped disc region, the linear phase ramp has an effect equivalent to an offset of the diffraction pattern at the detection plane. For small propagation distances, this offset is small, and as propagation distance increases, the offset effect of the phase ramp becomes more pronounced and therefore has a greater effect on the reconstruction.

At shorter propagation distances, it is common for the algorithm to construct the phase ramp in the region outside of the disc first of all, leaving a blurred disc with a flat surface in the centre. This process generally introduces a number of artefacts in the reconstruction at the disc edges (Fig. 2). It is possible that the order in which the diffraction patterns are considered plays a role in the formation of this object. With enough iterations, ptychography is able to overcome this initial incorrect object estimate, and reconstruct the correct low-frequency phase object.

\section{References:}

[1] AM Maiden et al, Scientific Reports 5 (2015), 14690.

[2] P Thibault et al, Nature 494 (2013), p. 68-71.

[3] AM Maiden \& JM Rodenburg, Ultramicroscopy 109 (2009), p. 1256-1262.

(a)

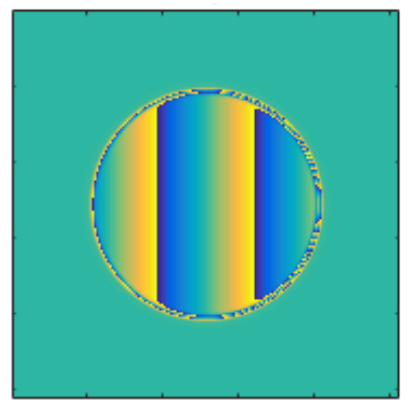

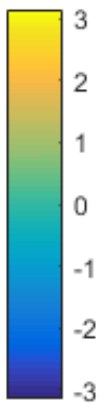

(b)

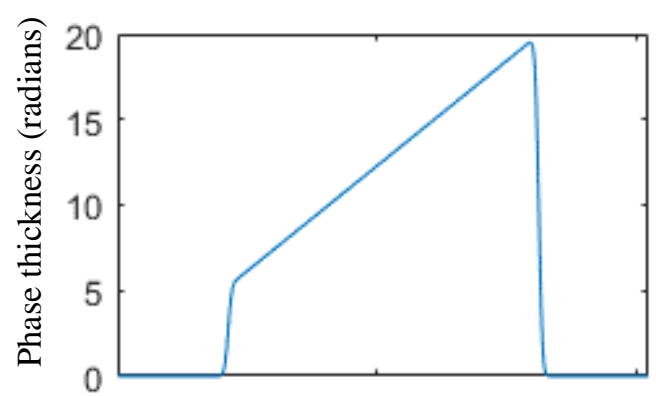

Figure 1. Phase image of the simulated object: (a) a phase-only soft-edged disc, diameter $820 \mathrm{~nm}$, with a linear ramp across its surface (scale bar in radians); and (b) a plot of its (unwrapped) phase profile.
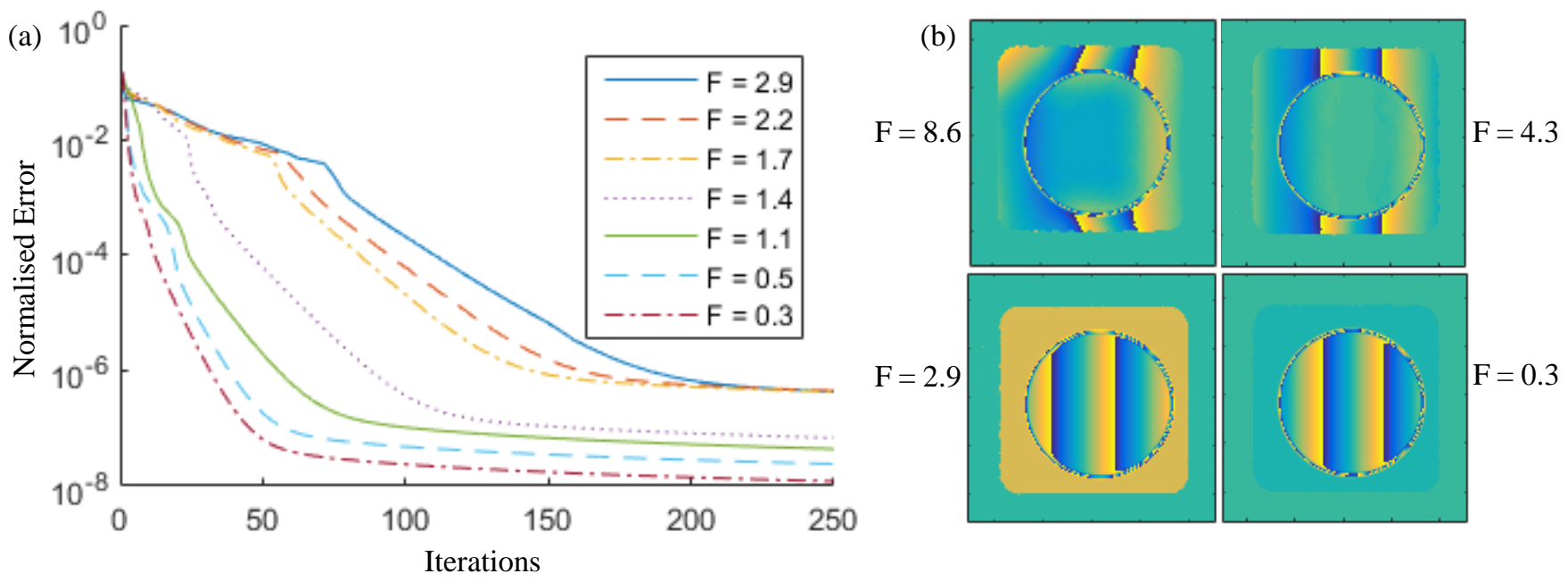

Figure 2. Results: (a) a graph displaying the normalised error versus number of iterations for experiments with a variety of Fresnel numbers; and (b) a comparison of object reconstructions after 500 iterations. Note the external phase ramp and artefacts at higher Fresnel numbers. 